http:// bjas.bu.edu.eg

\title{
Neonatal Anaesthesia
}

E.F.Gad Alla, M.A.khashaba and R.E.El- Sadek

Anesthesia and Intensive Care Dept., Faculty of Medicine, Benha Univ., Benha, Egypt

E-Mail : raniasharaf2@gmail.com

\begin{abstract}
Safe anaesthesia for neonates is based on understanding their unique physiology and response to medications so as best to provide analgesia and amnesia, depress stress responses, maintain cardiovascular stability, and return them to baseline status. Medications administered by any route have a similarly rapid uptake followed by the slower elimination phase as adults. However, the duration of these phases is altered by changes in body composition, protein binding, and maturation of organ function. To review all the recent guide lines in the anaesthetic management of neonates to provide the anesthesiologist with an understanding of the basics, clinical aspects and recent advances in the anaesthetic management of neonates. Surgical procedures in the first month also are considered emergent, or at least urgent, surgery. The most frequent surgical procedures in the first month are exploratory laparotomy for NEC, inguinal hernia repair, correction of pyloric stenosis, patent ductus arteriosus [PDA] ligation, a shunt procedure for hydrocephalus, and placement of a central venous catheter.
\end{abstract}

\section{Introduction}

Safe anaesthesia for neonates is based on understanding their unique physiology and response to medications so as best to provide analgesia and amnesia, depress stress responses, maintain cardiovascular stability, and return them to baseline status. Medications administered by any route have a similarly rapid uptake followed by the slower elimination phase as adults. However, the duration of these phases is altered by changes in body composition, protein binding, and maturation of organ function [1].

Neonatal anaesthetic care begins with a careful preoperative evaluation, including maternal history [medications, drug use, diabetes, etc] and birth history [small, appropriate, or large for gestational age, birth trauma, meconium, respiratory status, focused airway examination, current medications, laboratory data, congenital anomalies, echocardiogram, etc] [2].

Monitoring must be appropriate for the procedure. If invasive arterial or central venous monitoring is indicated, these need to be placed either by the anesthesiologist or the surgeon. One should never feel pressed to move forward without adequate monitoring [2].

There are Specific Neonatal Pathologies

Tracheo-oesophageal fistula/oesophageal atresia: it is a relatively common congenital malformation occurring in live births Commonly TEF is of 5 types [A-E], with type $\mathrm{C}$ being the most common [3].

Pyloric stenosis: Symptoms are apparent from 2nd to 6thweek of life. Neonates have severe projectile nonbilious vomiting with resultant hypochloremic dehydration [4].

Congenital diaphragmatic hernia: In most cases the aetiology remains unknown. A defect in the diaphragm, usually on the left side, results in herniation of midgut structures into the thoracic cavity [5].

Gastroschisis and exomphalos [omphalocele]: they are both ventral wall defects resulting in herniation of abdominal viscera. Diagnosis is ideally made on antenatal ultrasound scan [6].

Decreased ventilatory control and hyporesponsiveness to hypoxia and hypercarbia may be potentiated by anesthetic agents. Hypothermia, anemia, and respiratory muscle fatigue may also play a role [7].

\section{Anaesthetic drugs in neonates}

The pharmacokinetics of drugs in neonates are different than in older children and adults. Factors affecting the metabolism of drugs in neonates include a larger volume of distribution, decreased protein binding, and decreased fat stores and immature renal and hepatic function. These physiological changes alter the amount of drugs used in neonates.

\subsection{Intravenous Agents \\ 2.1.1 Sedative/Hypnotics}

The common sedative hypnotics used in neonates include propofol, thiopental, and ketamine.

\subsubsection{Thiopental}

Because of the large volume of distribution in neonates, it may be necessary to use large doses of thiopental for induction of anesthesia. However, because of their reduced clearance, the effect may last longer than anticipated. Thiopental can cause hypotension in neonates who are volume-depleted, especially in infants who are scheduled for emergency surgery [8].

\subsubsection{Propofol}

It is a phenyl sedative hypnotic, is a commonly used induction agent in infants and children. In a randomized trial comparing intravenous propofol with atropine, succinylcholine, and morphine, it was noted that propofol maintained adequate hemodynamics in neonates [9].

\subsubsection{Ketamine}

Ketamine, an N-methyl-D-aspartic acid [NMDA] antagonist, is used for induction of anesthesia in neonates who may have cardiovascular instability. Ketamine is still used frequently in neonates with congenital heart disease for induction of anaesthesia [8].

\subsubsection{Opioids}

Opioids are used extensively in the management of anesthesia in neonates.. Infants who are on long-term doses of opioids may develop tolerance and may have to be placed on methadone, a longer-acting opioid [10]. 


\subsubsection{Fentanyl}

This synthetic opioid is commonly used for sedation in the neonate in the intensive care unit as well as in the operating room. The use of fentanyl in association with benzodiazepines may lead to hypotension and hemodynamic instability [11].

\subsection{Morphine}

Morphine is used frequently in the intensive care unit for postoperative pain control. Morphine infusions in the perioperative period have resulted in minor prolongation of postoperative ventilation. [12].

\subsubsection{Neuromuscular blocking agents}

Neuromuscular blocking agents [NMBAs] are frequently used during neonatal anaesthesia to facilitate tracheal intubation, assist with controlled ventilation, relax abdominal musculature, and ensure immobility. Factors that influence the choice of agent include the time of onset, duration of action, cardiovascular effects, and mechanism of clearance/elimination [13].

\subsubsection{Succinylcholine}

Caution should be exercised when administering a second dose of succinylcholine because this can lead to vagally mediated bradycardia or sinus arrest. Pretreatment with atropine is recommended [14].

\section{Anesthetic management of the neonate}

Effective evaluation, preparation, and anesthetic management of the neonate depend on appropriate knowledge, clinical skills, and vigilance by the anesthesiologist. For safe and effective care, the anesthesiologist must take extraordinary care to understand the current status of the patient, the nature of the planned surgery, and the potential need for stabilization and preparation before surgery. After ensuring that the patient has been adequately prepared, the anesthesiologist needs to develop a detailed plan that encompasses the issues of anesthetic equipment and monitoring, airway management, drug choice, fluid management, temperature control, anticipated surgical needs, pain management, and postoperative care [15].

\subsection{Preanesthetic evaluation physical examination}

Physical examination of the newborn is focused by the condition requiring surgical intervention. Hydration is often an important issue because of both fasting and losses related to the surgical lesion. Clinical signs of dehydration include a sunken fontanelle, poor skin turgor, dry mucus membranes, sunken eyes, poor skin perfusion, delayed capillary refill, hypothermia, and a history of tachycardia or absent urine output. If there are clinical signs of dehydration, efforts should be made to correct the deficits before surgery, except in extreme, life-threatening situations [16].

\subsection{Preanesthetic evaluation-laboratory}

Most laboratory investigations related to the underlying surgical condition, such as radiologic investigations, computed tomography or magnetic resonance imaging studies, and echocardiography. However, most newborns will have, at a minimum, blood count and glucose level drawn. The hemoglobin in a newborn is primarily fetal hemoglobin, which has a higher affinity for oxygen than adult hemoglobin. Because of this higher affinity, the hemoglobin dissociation curve is shifted to the left, releasing less oxygen to the tissues than adult hemoglobin. [16].

Coagulation parameters are different in newborns compared with adults. Although platelet counts in term newborns are usually similar to adult values, lower values are frequently seen in the preterm. Unexplained thrombocytopenia can be an early sign of sepsis, and a falling count should be an impetus to look for other signs of sepsis. [17].

\subsection{Preanesthetic Plan}

The anesthesiologist has a host of anesthetic techniques from which to choose and can tailor the anesthetic to the requirements of the surgery and the condition of the neonate. Major factors that should be considered in planning the anesthetic include:

1- The need to have blood and blood products available before beginning the case.

2- The need for invasive monitoring.

3- The need for additional equipment for securing the airway or establishing vascular access.

4- The need to transport the child to and from the operating room.

5- The likelihood of postoperative ventilation.

6- The plan for postoperative pain relief [17].

Both the medical status of the patient and the planned surgical procedure will impact this planning. The anesthesiologist has the responsibility of clarifying any medical issues with the neonatologist before finalizing the plan, as well as clarifying any issues relates to the planned procedure with the surgeon. Occasionally, as planning progresses, it becomes obvious that the patient needs further medical resuscitation or evaluation before it is prudent to proceed with the procedure [18].

\subsection{Premedication}

Premedication is not commonly used for neonatal anesthetics. Sedation is not usually appropriate, and analgesics are rarely indicated before taking the patient to the operating room. In the past, premedication with atropine was occasionally used, especially for older neonates, above a month of age, where an inhalation induction was considered. Because of the dominance of the parasympathetic nervous system, bradycardia on induction or in response to inhalation agents is of concern [19]

\subsection{Intraoperative Considerations 3.5.1 Monitoring}

Physical observation of the patient is important in preanesthetic evaluation; it is difficult to use this monitor during a surgical procedure. Observation of the patient's color, capillary refill, warmth of skin, muscle tone, fullness of fontanelle, and chest expansion are useful monitors, but they are difficult to reliably observe once 
the patient is covered with surgical drapes. There is a large dependence on electronic monitors during the majority of the procedure. However, it should be remembered that heart and breath sounds heard through a precordial or esophageal stethoscope, the compliance determined during hand ventilation, the appearance of bleeding in the surgical field, and trends noted in the anesthetic record are all important observations that the anesthesiologist can use as part of the overall assessment of the patient [20].

\subsubsection{Temperature Control and Thermogenesis}

The newborn is at risk for significant metabolic derangements caused by hypothermia. Newborns, and especially preterms, do not have the normal compensatory mechanisms that infants and children have when exposed to a cold environment. The newborn does not shiver, increase activity, or effectively vasoconstrict like older children or adults do in response to cold. In addition, the newborn has a larger body surface area-toweight ratio that promotes heat loss, as well as low levels of subcutaneous fat for insulation. The primary mechanism the newborn has to respond to heat loss is nonshivering thermogenesis [21].

\subsubsection{Anesthetic Systems}

There is a long tradition in pediatric anesthesia of using semiopen, nonrebreathing systems for general anesthesia in newborns. Circuits such as the JacksonRees adaptation of the Ayre's T-piece and the Bain circuit have been the most commonly used in the United States. These and related circuits have the advantage of lightweight, easy-to-open valves or lack of valves, rapid changes in anesthetic concentration, minimal work of breathing, and high circuit compliance. [22].

\subsubsection{Induction of anesthesia}

There is no one method of induction and maintenance of anesthesia that is best for all patients. The current medical status of the patient, the surgical condition, the presence of ongoing fluid or blood losses, the gestational age of the patient, recent fasting, and the experience of the anesthesiologist are all important considerations. Most neonates who come to the operating room will have vascular access already established; if not, the first task before induction is to establish adequate vascular access after applying monitors. Although it may rarely be appropriate to use an inhalational induction if vascular access is difficult in the older newborn, near a month of age, it is mandatory to establish access first in the newborn that is preterm, medically unstable, has a full stomach, has a potentially difficult airway, or has ongoing fluid losses. [23].

\subsubsection{Regional anesthesia}

Regional anesthesia can be provided as a sole anesthetic or in combination with general anesthesia. The use of sole regional anesthesia in neonates and infants is provided for the ex-premature infant with a potential for apnea. For patients receiving combined general and regional anesthesia, early extubation is possible because the addition of regional anesthetic techniques eliminates the need for intraoperative narcotics in neonates, reduces or eliminates the need for muscle relaxants, and reduces the concentration of volatile agents needed for relaxation [24].

\subsubsection{Caudal block}

Caudal epidural block is frequently used for abdominal surgery in neonates and is probably the most commonly used regional anesthetic technique in neonates and infants. There are several different techniques described for performing a caudal. The landmarks are the coccyx, the two sacral cornua, and the posterior superior iliac spines [25].

\subsubsection{Epidural analgesia}

With the introduction of newer and smaller needles and epidural catheters we are now able to provide epidural analgesia in neonates and infants. Although most practitioners prefer using a caudal route to place catheters in the epidural space, we believe that with the introduction of ultrasound guidance, we are able to place lumbar catheters in neonates and infants easily. It is imperative to limit the dose of local anesthetic solution in neonates and children to avoid toxicity [26].

\subsubsection{Peripheral nerve blocks}

Common peripheral nerve blocks in neonates include penile blocks, ilioinguinal nerve blocks, lateral femoral cutaneous blocks, brachial plexus blocks, and head and neck blocks for neurosurgical procedures [27].

\subsubsection{Postoperative pain management}

The concepts of postoperative pain management are well known to most anesthesiologists. The use of intraoperative epidural anesthesia followed by postoperative epidural local anesthetics or opioids has been popular in older children and adults, and these techniques are being applied to neonates. In addition, most neonatologists are experienced with the intravenous administration of opioids for patient comfort. Each technique has its own risks and benefits. [28].

\subsubsection{Intravenous analgesia}

Opioids are the mainstay of analgesia in neonates and infants in the postoperative period. Morphine and fentanyl are frequently used in the neonatal intensive care unit for analgesia. However, the potential for opioid tolerance after prolonged infusion of opioids is not uncommon. To decrease the likelihood of opioid tolerance, one can rotate opioids or add other medications including continuous intravenous naloxone and intravenous methadone. Intravenous ketorolac, a nonsteroidal anti-inflammatory drug, has been used successfully in neonates and infants for pain control [29].

\subsubsection{Postoperative ventilation}

The choice of an anesthetic drug should be guided by the need for postoperative management of ventilation, as well as the drug's effects on the circulation and other organs. If the surgical procedure or the neonate's 
condition is such that postoperative ventilation is likely, the prolonged respiratory effects of opioids or any other drug are of little concern. However, if the surgical procedure is relatively short and by itself does not require postoperative ventilation, the clinician should carefully select drugs, as well as doses of anesthetic drugs and relaxants, that will not necessitate prolonged postoperative ventilation or intubation [30].

\section{Special considerations}

\subsection{Respiratory distress syndrome}

Because of the enormous technical ability of the neonatologist and the resources of neonatal intensive care units, many small infants survive and some need surgery. One of the frequent problems of preterm infants is the occurrence of the RDS secondary to a deficiency of surfactant. As discussed earlier, the use of exogenous surfactant has been widely used in premature infants of low birth weight either to prevent or to treat RDS. As a result, fewer infants now die of this entity, and the incidence of complications related to RDS has dropped [31].

Anesthetic concerns in these patients include evaluation of baseline oxygenation and potential presence of active bronchoconstriction. These patients often benefit from additional bronchodilator before induction. The baseline measure of oxygenation is important because these patients have less pulmonary oxygen reserve and may desaturate quickly with induction of anesthesia and hypoventilation. In patients with severe bronchopulmonary dysplasia, ventilatory management may be complicated by poor lung compliance and hyperinflation, as well as reactive airway disease. Although postanesthetic intubation is not usually required, a high index of suspicion should be used if there is significant clinical evidence of poor lung function preoperatively [31].

\subsection{Postoperative apnea}

Apnea and bradycardia are well-recognized, major complications during and after surgery in neonates. The infants at highest risk are those born prematurely, those with multiple congenital anomalies, those with a history of apnea and bradycardia, and those with chronic lung disease. The etiology of neonatal apnea is multifactorial. Decreased ventilatory control and hyporesponsiveness to hypoxia and hypercarbia may be potentiated by anesthetic agents [32].

Respiratory muscle fatigue may also play a role because neonates have a smaller percentage of type I fibers in their diaphragm and intercostal muscles. In addition, hypothermia and anemia can also contribute to the development of postoperative apnea [30].

The treatment of postoperative apnea or bradycardia may be as simple as tactile stimulation. However, some infants require mask ventilation or even prolonged intubation and ventilatory support. Infants with lifethreatening apnea and bradycardia before surgery may be receiving CNS stimulants. Caffeine and theophylline [metabolized to caffeine] act by increasing central respiratory drive and lowering the threshold of response to hypercarbia, as well as stimulating contractility in the diaphragm [33].

\subsection{Surgical procedures in neonates}

Surgical procedures in neonates are functionally divided into two periods: those performed in the first week and those performed in the first month. There has been a strong trend in recent years to put on emphasis of presurgical stabilization before taking the newborn to the operating room. This has reduced the emergent nature of newborn surgeries [34].

\subsection{Tracheoesophageal fistula}

The treatment of esophageal atresia and TOF can be both challenging and satisfying for the anesthesiologist. Death in the perioperative period typically results from prematurity or from an associated congenital heart defect. TOF occurs in approximately 1 in 3,000 live births. Approximately $85 \%$ consist of a fistula from the distal trachea to the esophagus and a blind proximal esophageal pouch. In $10 \%$ of cases, there is a blind proximal esophageal pouch with no TOF [35].

\subsection{Anesthetic considerations}

The repair of TOF can be done in the conventional method or by a thoracoscopic method. Both methods and the anesthetic implications for each technique will be described here. The presence of a gastrostomy reduces the potential for reflux of gastric juice during the surgical procedure. If a gastrostomy is present, the gastrostomy tube should be open to air and left at the head of the table under the anesthesiologist's observation to avoid kinking and obstruction [35].

\subsection{Pyloric stenosis}

Pyloric stenosis is a relatively frequent surgical disease of the neonate and infant. It can appear as early as the second week of life. The pathologic characteristics include hypertrophy of the pyloric smooth muscle with edema of the pyloric mucosa and submucosa. This process, which develops over a period of days to weeks, leads to progressive obstruction of the pyloric valve, causing persistent vomiting [36].

\subsection{Anesthetic management}

It is prudent to pass a large orogastric tube and aspirate the stomach contents because of the significant volume that may be present. This procedure greatly reduces the quantity of gastric fluid. A rapid-sequence induction is advisable because of the potential for additional volume in the stomach. Although awake intubation had been popular with some clinicians in the past, it is associated with a higher incidence of complications and is traumatic to the child [37].

Remifentanil is appropriate for opioid-based anaesthesia in paediatric patient because it provides haemodynamic stability and rapid recovery characteristics with minimal PO side effects [38].

Controlled ventilation reduces or eliminates the need for muscle relaxants for this surgery. At the end of the 
case, the patient should be wide awake before extubation [39].

\section{Summary}

In utero, oxygenated placental blood is divided to pass through either the liver or to the inferior vena cava via the ductus venosus, This oxygenated blood from the inferior vena cave preferentially streams across the right atrium through the foramen ovale into the left atrium. This blood passes through the left ventricle and aorta to supply the myocardium, head, and upper torso. Deoxygenated blood returns to the right atrium via the superior and inferior vena cava and is pumped out into the pulmonary arteries via the right ventricle.

The pulmonary system develops rapidly during the last trimester, with important changes in both the number of alveoli and the maturity of the pulmonary vascular system. These systems have not matured enough to provide adequate gas exchange until about 24 to 26 weeks gestation.

The pharmacokinetics of drugs in neonates are different than in older children and adults. Factors affecting the metabolism of drugs in neonates include a larger volume of distribution, decreased protein binding, and decreased fat stores and immature renal and hepatic function.

There is no one method of induction and maintenance of anesthesia that is best for all patients. The current medical status of the patient, the surgical condition, the presence of ongoing fluid or blood losses, the gestational age of the patient, recent fasting, and the experience of the anesthesiologist are all important considerations. Most neonates who come to the operating room will have vascular access already established; if not, the first task before induction is to establish adequate vascular access after applying monitors. Although it may rarely be appropriate to use an inhalational induction if vascular access is difficult in the older newborn, near a month of age, it is mandatory to establish access first in the newborn who is preterm, medically unstable, has a full stomach, has a potentially difficult airway, or has ongoing fluid losses.

The choice of an anesthetic drug should be guided by the need for postoperative management of ventilation, as well as the drug's effects on the circulation and other organs. If the surgical procedure or the neonate's condition is such that postoperative ventilation is likely, the prolonged respiratory effects of opioids or any other drug are of little concern. However, if the surgical procedure is relatively short and by itself does not require postoperative ventilation, the clinician should carefully select drugs, as well as doses of anesthetic drugs and relaxants, that will not necessitate prolonged postoperative ventilation or intubation.

The most frequent major surgical procedures performed in the first week of life are for congenital diaphragmatic hernia $[\mathrm{CDH}]$, omphalocele and gastroschisis, tracheoesophageal fistula [TOF], intestinal obstruction, and meningomyelocele. Some of these conditions, such as $\mathrm{CDH}$, omphalocele and gastroschisis, and meningomyelocele, are obvious at birth. It may take hours or days for a TOF or intestinal obstruction to become manifest.

Surgical procedures in the first month also are considered emergent, or at least urgent, surgery. The most frequent surgical procedures in the first month are exploratory laparotomy for NEC, inguinal hernia repair, correction of pyloric stenosis, patent ductus arteriosus [PDA] ligation, a shunt procedure for hydrocephalus, and placement of a central venous catheter.

\section{References}

[1] GM. Pacifci, Pharmacokinetics of antivirals in neonate.Early Hum.Dev, Vol. 81, PP. 773-80, 2005.

[2] K. Konig, DM. Casalaz, EJ. Burke, \& A. Watkins, Accuracy of noninvasive blood pressure monitoring in very preterm infants.IntensCare Med, Vol. 38, PP. 670, 2012.

[3] G. Kinottenbelt, A. Skinner \& C. Seefelder, Tracheoesophageal fistula TOF and oesophageal atresia OA Best Pract Res ClinAnaesthesiol, Vol. 24, PP. 387-401, 2010.

[4] KA. Haselby, SF. Dierdorf, G. Krishna, CC. Rao, TM. Wolfe, \& WL. McNiece, Anaesthetic implications of neonatal necrotizing enterocolitis. Can AnaesthSoc J, Vol. 29, PP. 255-9, 1982.

[5] P. Bérémieux, T. Gaillot, A. de la Pintière, A. Beuchee, L. Pasquier, \& E. Habonimann, et al. Congenital diaphragmatic hernia: Prenatal diagnosis permits immediate intensive care with high survival rate in isolated cases.A population-based study. PrenatDiagn, Vol. 24, PP. 487-93, 2004.

[6] SO. Ekenze, PA. Anyanwu \& UO. Ezomike,.Profle of pediatric abdominal surgical emergencies in a developing countries.Int Surg. Oct-Dec, Vol. 95, (4), PP. 319-24, 2010.

[7] L. Welborn, 2002. Perioperative Management of the Former Preterm Infant in PediatricAnesthesia Handbook. Terrance A. Yemen, editor. New York: McGraw Hill, Vol. 130, PP. 142

[8] P. Asadi, H.B. Ghafouri, M. Yasinzadeh, S.M. Kasnavieh, and E. Modirian, Ketamine and atropine for pediatric sedation: a prospective double-blind randomized controlled trial. Pediatr Emerg Care, Vol. 29, PP. 136-139, 2013.

[9] V. Chidambaran, A. Costandi, and A. D'Mello, Propofol: a review of its role in pediatric anesthesia and sedation. CNS Drugs, Vol. 29, PP. 543-563, 2015.

[10] J. Hannan, and Anderson B: Pharmacodynamic interaction models in pediatric anesthesia. Paediatr Anaesth, Vol. 25, PP. 970-980, 2015.

[11] A.M. Cressman, G. Mazereeuw, Q. Guan, W. Jia, T. Gomes, and D.N. Juurlink, Availability of naloxone in Canadian pharmacies: A population-based survey. CMAJ Open, Vol. 5, PP. E779-E784, 2017.

[12] MA. Baarslag, K. Allegaert, JN. Van Den Anker, Paracetamol and morphine for infant and neonatal pain; still a long way to go? Expert Rev Clin Pharmacol, Vol. 10, PP. 111-126, 2017. 
[13] A.R. Wolf, Ductal ligation in the very low-birth weight infant: simple anesthesia or extreme art? Paediatr Anaesth, Vol. 22, PP. 558-563, 2012.

[14] V. Luce, H. Harkouk, C. Brasher, D. Michelet, J. Hilly, M. Maesani, et al. Supraglottic airway devices vs tracheal intubation in children: a quantitative meta-analysis of respiratory complications. Paediatr Anaesth, Vol. 24, PP. 1088-1098, 2014.

[15] Imdad et al, Imdad A, Bautista RM, Senen KA, et al: Umbilical cord antiseptics for preventing sepsis and death among newborns. Cochrane Database Syst Rev, 2013.

[16] W. Benitz, Committee on Fetus and Newborn. Policy statement: hospital stay for healthy term newborns. Pediatrics, Vol. 135, PP. 948-953, 2015.

[17] W. Mahle, G. Martin, R. Beekman, Endorsement of health and human services recommendation for pulse oximetry screening for critical congenital heart disease. Pediatrics, Vol. 129, PP. 190, 2012.

[18] M. Wenner Van Vleet, General care of the newborn. In eds: , ed 2. Heidelberg: Springer, PP. 137-158, 2012.

[19] T.M. Wani, M. Rafiq, N. Akhter, Upper airway in infants-a computed tomography-based analysis. Paediatr Anaesth, 2017.

[20] S.B. Hooper, A.B. Te Pas, and M.J. Kitchen, Respiratory transition in the newborn: a three-phase process. Arch Dis Child Fetal Neonatal Ed, 2016.

[21] A. Alvik A, O.O. Aalen, and R. Lindemann, Early fetal binge alcohol exposure predicts high behavioral symptom scores in 5.5-year-old children. Alcohol. Clin. Exp. Res, Vol. 37, PP. 1954-1962, 2013.

[22] R.C. Brockwell, and J.J. Andrews, Inhaled anesthetic delivery systems. In Miller R.D. eds: Miller's Anesthesia, 7th ed. Philadelphia: Churchill Livingstone, PP. 667-718, 2010.

[23] S. Ryan, and C.J. Neilsen, Global warming potential of inhaled anesthetics: application to clinical use. Anesth Analg, Vol. 111, PP. 92-98, 2010.

[24] G, Frawley, G. Bell, N. Disma, Predictors of failure of awake regional anesthesia for neonatal hernia repair: data from the General Anesthesia compared to Spinal anesthesia GAS study: comparing apnoea and neurodevelopmental outcomes. Anesthesiology, Vol. 123, PP. 55-65, 2015.

[25] LJ. Jones, PD. Craven, A. Lakkundi, Regional spinal, epidural, caudal versus general anesthesia in preterm infants undergoing inguinal herniorrhaphy in early infancy. Cochrane Database Syst Rev, 2015.

[26] AJ. Davidson, N. Disma, JC. de Graaff, Neurodevelopmental outcome at two years of age after general and awake-regional anaesthesia in infancy: a randomized controlled trial. Lancet, Vol. 387, PP. 239-250, 2016.
[27] M.F. Mulroy, R.S. Weller, and G.A. Liguori, A checklist for performing regional nerve blocks. Reg Anesth Pain Med, Vol. 39, PP. 195-199, 2014.

[28] A. Alam, T. Gomes, H. Zheng, M.M. Mamdani, D.N. Juurlink, and C.M. Bell, Long-term analgesic use after low-risk surgery: A retrospective cohort study. Arch Intern Med, Vol. 172, PP. 425-430, 2012.

[29] D. Anghelescu, L. Faughnan, L. Oakes, K. Windsor, and D. Pei, Parent-controlled PCA for pain management in pediatric oncology: Is it safe? J Pediatr Hematol Oncol, Vol. 34, PP. 416-420, 2013.

[30] AJ. Davidson, N. Disma, JC. de Graaff, Neurodevelopmental outcome at two years of age after general and awake-regional anaesthesia in infancy: a randomized controlled trial. Lancet, Vol. 387, PP. 239-250, 2016.

[31] I. Ceelie, SN. de Wildt, M. van Dijk, Effect of intravenous paracetamol on postoperative morphine requirements in neonates and infants undergoing major noncardiac surgery. JAMA, Vol. 309, PP. 149-154, 2013.

[32] K. Coleman, ND. Robertson, GA. Dissen, Isoflurane anesthesia has long-term consequences on motor and behavioral development in infant rhesus macaques. Anesthesiology, Vol. 126, PP. 74-84, 2017.

[33] L. Sun, and Y. Wang, Demographic and perinatal outcome data of fetuses with SUA/PRUV. J Matern Fetal Neonatal Med, Vol. 1-6, 2017.

[34] FC. Brunicardi, DK. Andersen, TR. Billiar, Schwartz's principles of surgery. New York: McGraw-Hill, 2015.

[35] Nazir Zafar, Arif Muhammad, Khan Mateen, and Qamar Javaria, Recurrent and acquired tracheoesophageal fistulae TEF-minimally invasive management. J Pediatr Surg, Vol. 52, PP. 16881690, 2017.

[36] A. Al-Ansari, and T.I. Altokhais, Recurrent pyloric stenosis. Pediatr Int Off J Jpn Pediatr Soc, Vol. 58, PP. 619-621, 2016.

[37] H. Louati, H. Zitouni, M. Belhajmansour, 2017: A difficult recurrent hypertrophic pyloric stenosis. J Neonatal Surg, Vol. 6, PP. 48, 2016.

[38] Hamid AMA, Shady AFA, Azeem ESA. Remifentanil infusion as a modality for opioidbased anaesthesia in paediatric practice. Indian $\mathrm{J}$ Anaesth 2010;54:318-23.

[39] A. Granstrom, S. Felder, and P. Frykman, Laparoscopic repyloromyotomy following open pyloromyotomy for recurrent pyloric stenosis: a case report and review of the literature. Eur $\mathrm{J}$ Pediatr Surg, Vol. 23, PP. 499-501, 2013. 